\title{
New ferromagnetic core shapes for induction sensors
}

\author{
C. Coillot ${ }^{1}$, J. Moutoussamy ${ }^{2}$, M. Boda ${ }^{3}$, and P. Leroy ${ }^{2}$ \\ ${ }^{1}$ Laboratoire Charles Coulomb, BioNanoNMRI group, University Montpellier II, Place Eugene Bataillon, \\ 34090 Montpellier, France \\ ${ }^{2}$ LPP/CNRS/UPMC/Ecole Polytechnique, Route de Saclay, 91128 Palaiseau, France \\ ${ }^{3}$ SubSeaStem, 25 rue des Ondes, 12000 Rodez, France \\ Correspondence to: C. Coillot (christophe.coillot@univ-montp2.fr)
}

Received: 10 August 2013 - Revised: 30 October 2013 - Accepted: 5 December 2013 - Published: 15 January 2014

\begin{abstract}
Induction sensors are used in a wide range of scientific and industrial applications. One way to improve these is rigorous modelling of the sensor combined with a low voltage and current input noise preamplifier aiming to optimize the whole induction magnetometer. In this paper, we explore another way, which consists in the use of original ferromagnetic core shapes of induction sensors, which bring substantial improvements. These new configurations are the cubic, orthogonal and coiled-core induction sensors. For each of them we give modelling elements and discuss their benefits and drawbacks with respect to a given noise-equivalent magnetic induction goal. Our discussion is supported by experimental results for the cubic and orthogonal configurations, while the coiled-core configuration remains open to experimental validation. The transposition of these induction sensor configurations to other magnetic sensors (fluxgate and giant magneto-impedance) is an exciting prospect of this work.
\end{abstract}

\section{Introduction}

The function of induction magnetometers is to measure extremely weak magnetic fields. Their field of application is very large and covers soil characterization for agriculture (Sudduth et al., 2001), earthquake survey or magnetotelluric waves observation, and natural electromagnetic waves near the surface of the Earth (lightning observations (Ozaki et al., 2012), whistlers (Lichtenberger et al., 2008)) or in space (Roux et al., 2007). In these applications the induction sensors must cover a wide frequency range from millihertz $(\mathrm{mHz})$, for magnetotelluric observations, up to megahertz $(\mathrm{MHz})$, for plasma waves observation. In order to remove the resonance of the induction sensor, they are combined either with a feedback flux (Seran and Fergeau, 2005) or a current amplifier (Prance et al., 2000). The extension of their frequency range is made possible using two windings on the same ferromagnetic core separated by a magnetic mutual reducer (Coillot et al., 2010), while a relevant solution, called the dual-resonant search coil, permits combination of the two windings into a single one (Ozaki et al., 2013). The design of such an instrument requires obtaining a reli- able modelling tool to match the measurement requirements. The main specification of the measurement is usually given in terms of noise-equivalent magnetic induction (NEMI in $T / \sqrt{\mathrm{Hz}}$ ) either at a given frequency or by its spectrum over a frequency range.

In previous works authors have focused on physical modelling of the induction sensor performances in terms of NEMI (Seran and Fergeau, 2005; Korepanov and Pronenko, 2010) or low-noise amplifier design (Rhouni, 2012; Shimin et al., 2013).

The appropriate core and coil parameters can be found by reformulating the problem as a mathematical optimization problem (Coillot et al., 2007; Yan, 2013). Some analytical formulae are proposed in order to dimension the system in very limited cases. In Grosz and Paperno (2012), the authors present the design of a low-frequency induction magnetometer, their assumption being that the impedance of the coil is simply equal to its resistance.

In this work we explore new tracks of improvement guided by modifications in the shape of the ferromagnetic core. The first solution is the cubic induction sensor inspired by Dupuis (2003), where the core consists of 12 rods assembled in a 
cubic configuration (which could be extended to an array). For a given measurement direction the coil is distributed on the four edges of the cube. In the following, we will present the orthogonal induction sensor, which consists in an helical ferromagnetic core which canalizes the magnetic flux. The magnetic flux through the core turns is then measured by a coil wound orthogonally to the direction of the external magnetic field. This allows for significant reduction of the number of coil turns and reduction of the resistance of the coil for a given flux. The third induction sensor is the coiled ferromagnetic wire core, where the core is assumed to be made with a coilable ferromagnetic wire.

\section{Induction sensor basics}

\subsection{Elements of the electrical model}

Induction sensors (Ripka, 2000; Tumanski, 2007) are classically built with an $N$-turns coil. According to Lenz's law, when the coil is immersed in a magnetic field, a voltage $e$ is induced.

The resistance of the coil $(R)$ can be approximately computed using the following formula:

$R=4 \rho N \frac{\left(d+N\left(d_{\mathrm{w}}+2 t\right)^{2} / L_{\mathrm{w}}\right)}{d_{\mathrm{w}}^{2}}$,

where $\rho$ is the material resistivity (copper or aluminium are usually preferred), $d$ is the internal diameter of the coil, $d_{\mathrm{w}}$ is the wire diameter, $L_{\mathrm{w}}$ is the length over which the winding is distributed and $t$ is the thickness of the wire insulator.

The voltage difference between turns and layers is associated with electrostatic energy storage. This is usually represented by a capacitance $C$ on the electrokinetic model. The computation of this capacitance depends on the winding strategy; one can notice that discontinuous winding should be preferred as to avoid parasitic resonances (Coillot and Leroy, 2012).

Optionally, the wire is coiled around a ferromagnetic core, taking advantage of its magnetic gain (Bozorth and Chapin, 1942), known as apparent permeability $\left(\mu_{\text {app }}\right)$ and given in Eq. (2):

$\mu_{\text {app }}=\frac{\mu_{\mathrm{r}}}{1+N_{z}\left(\mu_{\mathrm{r}}-1\right)}$,

where $\mu_{\mathrm{r}}$ is the relative permeability and $N_{z}$ is the magnetometric demagnetizing coefficient in the $z$ direction. For a long cylinder core (i.e. length-to-diameter ratio: $m=L_{\mathrm{c}} / d \gg$ 1), the approximation of ellipsoid demagnetizing coefficient given in Osborn (1945) is valid:

$N_{z}(m)=\frac{1}{m^{2}}(\ln (2 m)-1)$.

When a "diabolo" core is used (Coillot et al., 2007) the apparent permeability is increased thanks to the magnetic flux

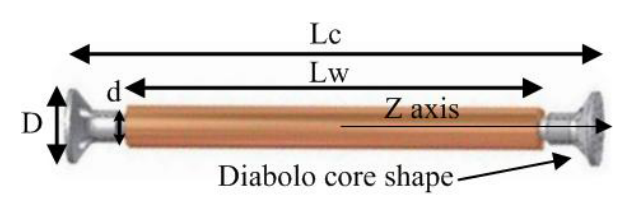

Figure 1. Diagram of an induction sensor with a diabolo core shape.

concentrators (shown in Fig. 1). In this work the various ferromagnetic core shapes will be linked to the one of the diabolo cores. In the case of diabolo core shape the apparent permeability equation becomes

$\mu_{\text {app-diab }}=\frac{\mu_{\mathrm{r}}}{1+N_{z}\left(m^{\prime}\right) \frac{d^{2}}{D^{2}}\left(\mu_{\mathrm{r}}-1\right)}$,

where $N_{z}\left(m^{\prime}\right)$ is the magnetometric demagnetizing coefficient for a cylinder of length-to-diameter ratio $m^{\prime}=L_{\mathrm{c}} / D$, while $d^{2} / D^{2}$ represents the surface ratio between the centre and the end surfaces of the core.

In the case of ferromagnetic core induction sensor, the inductance equation given in Tumanski (2007) is recalled here:

$\mathrm{€}=\lambda N^{2} \mu_{0} \frac{\mu_{\mathrm{app}} S}{L_{\mathrm{c}}}$,

where $S$ is the ferromagnetic core section, $\mu_{0}$ is the vacuum permeability and $\lambda=\left(L_{\mathrm{c}} / L_{\mathrm{w}}\right)^{2 / 5}$ is a correction factor proposed in Lukoschus (1979).

\subsection{Transfer function of the induction sensor}

Using a ferromagnetic core exhibiting an apparent permeability $\mu_{\text {app }}$, the induced voltage is expressed (in harmonic regime at the pulsation $\omega$ ) as

$e=-j \omega N S \mu_{\text {app }} B$,

where $j$ represents the unit imaginary number $j^{2}=-1$ and $B$ is the magnetic flux density. The electrokinetic modelling assumes that the induced voltage is in series with the resistance and the inductance, while the accessible voltage $(V)$ is measured at the capacitance terminals. Thus, the transmittance $(T(j \omega))$ is given by the following equation:

$T(j \omega)=\frac{V}{B}=\frac{-j \omega N S \mu_{\mathrm{app}}}{1-L C \omega^{2}+j R C \omega}$.

This transmittance exhibits a resonance at pulsation $\omega_{0}=$ $1 / \sqrt{(L C)}$. Beyond the resonance the induced voltage will decrease. The very high value of the induced voltage at the resonance frequency can be useful for some applications, while it should be removed and the transfer function must be flattened in induction magnetometer applications requiring wide-band measurements. This is typically true for applications where 
natural electromagnetic waves are measured, such as earthquake measurements, whistler observations and space plasmas. In such applications two kinds of electronic conditioners are classically implemented: feedback flux amplifiers or current amplifiers. In both cases, the transfer function will be flattened over about 3 to $\sim 6$ decades.

\subsection{Noise-equivalent magnetic induction}

Noise-equivalent magnetic induction (NEMI), expressed in $\frac{T}{\sqrt{(\mathrm{Hz})}}$, is the relevant quantity to determine the ability of the magnetometer to measure weak magnetic fields. The NEMI is defined as the square root of the total power spectrum density of the input reffered noise (PSD INPUT $_{\text {) related to the }}$ transfer function modulus $(T(j \omega))$ :

$\mathrm{NEMI}=\sqrt{\frac{\text { PSD }_{\text {INPUT }}}{\left|T(j \omega)^{2}\right|}}$,

where

$\mathrm{PSD}_{\mathrm{INPUT}}=4 k T R+e_{\mathrm{PA}}^{2}+\left(Z i_{\mathrm{PA}}\right)^{2}$,

where $k$ is the Boltzmann constant, $T$ is the temperature, $Z$ is the impedance and the electronic amplifier noise parameters are $e_{\mathrm{PA}}=4 \mathrm{nV} / \sqrt{(\mathrm{Hz})}$ and $i_{\mathrm{PA}}<20 \mathrm{fA} / \sqrt{(\mathrm{Hz})}$. Due to the low-frequency context, the current noise contribution (i.e. $\left.\left(Z i_{\mathrm{PA}}\right)^{2} \ll e_{\mathrm{PA}}\right)$ will be neglected.

\section{Cubic induction sensor}

\subsection{Description of the sensor configuration}

The cubic magnetometer proposed in Dupuis (2003) combines multiple induction sensors to form a cubic array (a diagram and a prototype are shown in Fig. 2). The advantages claimed by the author are the increase in sensitivity and the reduction of the self-inductance since the required turn number can be dispatched between the different edges. The cores are implemented in such a way that they are not coupled on the magnetic point of view but are connected in series on the electrical point of view. The sensitivity benefit is related to an increase in the apparent permeability. Our current goal is to confirm the behaviour of this original induction sensor configuration, briefly presented in Coillot and Leroy (2012), through experimental measurements with a prototype and the validation of the apparent permeability equation with the aim of comparison to classical induction sensors.

The edges of the cubic induction sensor are constituted by cylinder ferromagnetic cores of length $L_{\mathrm{c}}$ and diameter $d$. Due to the cubic shape, the demagnetizing coefficients are the same in the three directions:

$N_{x}=N_{y}=N_{z}=\frac{1}{3}$.
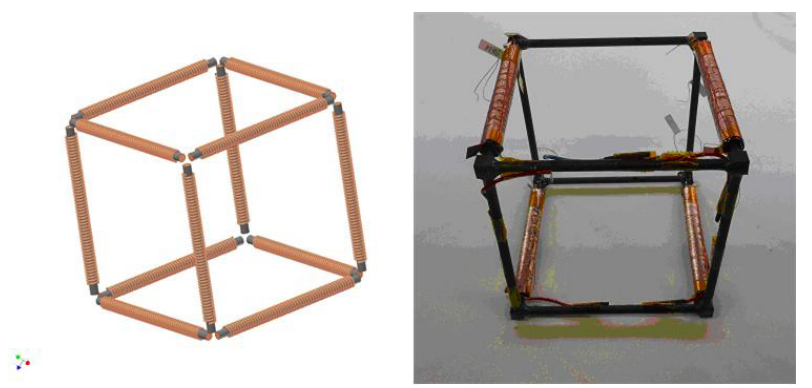

Figure 2. Cubic induction sensor diagram (left picture) and $85 \mathrm{~cm} \times 85 \mathrm{~cm} \times 85 \mathrm{~cm}$ prototype (right picture).

The flux caught by the square area face is distributed between the four ferromagnetic cores with a ratio corresponding to the surface ratio. The equation of the apparent permeability of the cubic sensor $\left(\mu_{\text {app-cub }}\right)$ is then obtained as a special case of the diabolo core apparent permeability formula (given by Eq. 4) where the demagnetizing coefficient is equal to $\frac{1}{3}$ and the surface ratio is $L_{\mathrm{c}}^{2} /\left(4 \times \pi d^{2} / 4\right)$; this leads to

$\mu_{\text {app-cub }}, y, z=\frac{\mu_{\mathrm{r}}}{1+N_{x, y, z}\left(\mu_{\mathrm{r}}-1\right) \frac{\pi d^{2}}{L_{\mathrm{c}}^{2}}}$.

For sufficiently high relative permeability (i.e. $\mu_{\mathrm{r}} \gg 1$ and $N \mu_{\mathrm{r}} \gg 1$ ), we can simply write

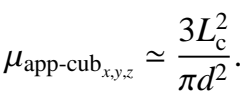

The previous formula is different from the one presented in Coillot and Leroy (2012), which is only valid for rods with square sections (despite what is claimed in the article).

\subsection{Experimental results}

A prototype cubic induction sensor has been built to evaluate the modelling of the apparent permeability and the impact on the resonance frequency. The design equations which have been used are directly derived from the one of the classical induction sensors presented above. The design parameters (turns number, core diameter, copper wire diameter) have been computed to allow the prototype to reach a NEMI value close to $0.7 \mathrm{pT} / \sqrt{\mathrm{Hz}}$ at $10 \mathrm{~Hz}$. The design parameters of the prototype are summarized in Table 1.

The cubic induction sensor prototype (left picture in Fig. 2) is a $85 \mathrm{~cm} \times 85 \mathrm{~cm} \times 85 \mathrm{~cm}$ cube. A ferrite cube has been mounted at each corner of the cube to ensure a closed ferromagnetic path. The ferromagnetic material used for the ferromagnetic parts is B1 ferrite material whose initial relative permeability is typically about 2500 . The ferromagnetic core has been wound in a single direction. In this direction, each of the four edges were wound with 8000 turns of $70 \mu \mathrm{m}$ diameter copper coil. Each coil has been connected in serial from the electrical point of view but in opposition from the 
Table 1. Design parameters of the cubic induction sensor prototype for a NEMI goal: $0.7 \mathrm{pT} / \sqrt{(\mathrm{Hz})}$ at $10 \mathrm{~Hz}$, assuming $e_{\mathrm{PA}}=$ $4 \mathrm{nV} / \sqrt{(\mathrm{Hz})}$ and $i_{\mathrm{PA}}=20 \mathrm{fA} / \sqrt{(\mathrm{Hz})}$.

\begin{tabular}{ll}
\hline Cylinder core length $\left(L_{\mathrm{c}}\right.$ in $\left.\mathrm{mm}\right)$ & 85 \\
\hline Core diameter $(d$ in $\mathrm{mm})$ & 4 \\
\hline Copper wire turns $(N$ per core $)$ & 8000 \\
\hline Wire diameter $\left(d_{\mathrm{w}}\right.$ in $\left.\mathrm{mm}\right)$ & 0.07 \\
\hline Layer number & $n_{\mathrm{l}}=4$ \\
\hline Winding length $\left(L_{\mathrm{w}}\right.$ in $\left.\mathrm{mm}\right)$ & 80 \\
\hline Resistance $(R$ in $\Omega)$ & 1300 \\
\hline Apparent permeability $\left(\mu_{\text {app-cub }}\right)$ & 354
\end{tabular}

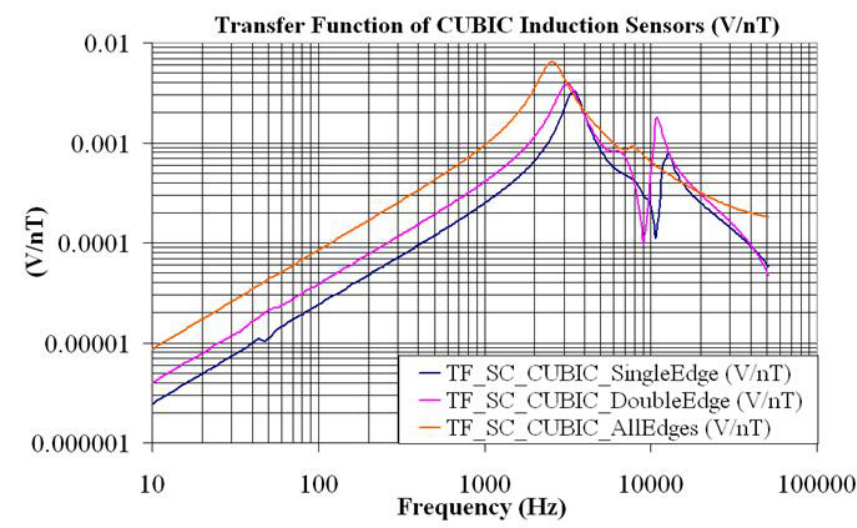

Figure 3. Transfer function of the cubic induction sensor: one edge (dark blue), two edges (pink) and four edges (orange curve).

magnetic field point of view in order to cancel the mutual induction between edges. It results that from a single edge to four edges, an increase in inductance by a ratio of 4 is expected (instead of 16 when inductances are magnetically coupled). The transfer function has been measured in three cases: single edge, double edges and four edges. This shows that the increase in gain (before the resonance) is proportional to the edge number and is multiplied by almost 4 from a single edge to four edges. The reason why the ratio between four edges and single edges is not precisely equal to 4 could be explained by a unbalanced magnetic path (namely the fluxes seen by each edge are not exactly identical). The resonance frequency varies weakly (from $3400 \mathrm{~Hz}$ for the single edge to $2700 \mathrm{~Hz}$ for four edges). This could be related to the fact that when the edges are connected together, the total inductance increases, while the total capacitance decreases; consequently the resonance frequency does not vary significantly. This property is of great interest for the design of wide-band and compact induction sensors. However, the multiple resonance next to the main resonance could make the use of feedback flux or current amplifiers difficult.
The apparent permeability deduced from the measurement (cf. Fig. 3) is $\mu_{\text {app-cub-meas }}=354$, while the numerical application of Eq. (11) gives a rather close approximation $\left(\mu_{\text {app-cub }}=368\right)$, which validates our modelling attempt.

\subsection{Discussion}

In order to evaluate the benefit of the cubic induction sensor, we will compare its apparent permeability and inductance to the one of a cylinder core induction sensor of the same length $\left(L_{\mathrm{c}}\right)$ and diameter $(d)$. Under the ellipsoid shape approximation, its demagnetizing coefficient is given by Eq. (3). Thus the long cylinder's apparent permeability (Eq. 2) can be written (assuming $\left.N_{z}(m) \mu_{\mathrm{r}} \gg 1\right)$

$\mu_{\text {app }}=\frac{1}{N_{z}(m)}=\frac{L_{\mathrm{c}}^{2}}{d^{2}} \frac{1}{\ln \left(2 \frac{L_{\mathrm{c}}}{d}\right)-1}$.

The comparison between the cubic sensor and the usual induction sensor can be done through the ratio between their apparent permeability (from Eqs. 12 and 13), which leads to

$\frac{\mu_{\text {app-cub }}}{\mu_{\text {app }}}=\frac{3}{\pi}\left(\ln \left(2 \frac{L_{\mathrm{c}}}{d}\right)-1\right)$.

This comparison suggests that the cubic induction sensor has a higher apparent permeability than the single rod since $\frac{L_{\mathrm{c}}}{d} \gg 1$. For example, for a $L_{\mathrm{c}} / d$ ratio equal to 10 , the cubic sensor apparent permeability will be about two times higher than the one of the single rod.

\section{Orthogonal induction sensor}

\subsection{Description of the sensor configuration}

In an orthogonal search coil, a helical core (shown in Fig. 4) is used to enhance the flux catched by each turn. Because of the two ferromagnetic discs (diameter $D$ ) mounted at the ends of the helicore (whose total length is $L_{\mathrm{c}}$ ), and assuming a high relative permeability $\left(\mu_{\mathrm{r}}\right)$, the external magnetic flux is canalized by the ends of the core and driven through each ferromagnetic core turn. The ferromagnetic turns are assumed to be square with side $d$.

To compute the induced voltage, we have to consider the angle between the normal vector of the coil turn section and the direction of the magnetic field inside the helicore; this angle $(\varphi)$, called the helix angle, is defined as

$\varphi=\arctan \left(\frac{b}{a}\right)$.

where the pitch of the helicore $(2 \pi b)$ is determined as

$2 \pi b=\frac{L_{\mathrm{w}}}{n}$,

where $L_{\mathrm{w}}$ is the length of the core on which the copper wire is wound and $2 a$ is the average diameter of the ferromagnetic core helix (cf. Fig. 4). 


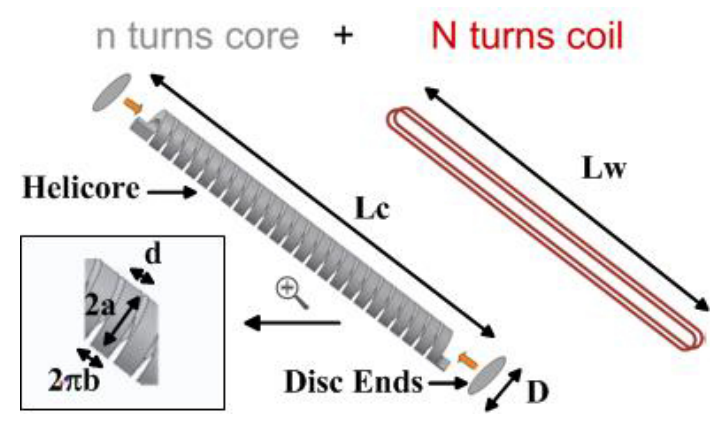

Figure 4. Diagram of the orthogonal induction sensor.

For $N$ turns of coil surrounding the $n$ core section, the induced voltage $\left(e_{\mathrm{hc}}\right)$ becomes

$e_{\mathrm{hc}}=-j \omega N n \mu_{\mathrm{app}-\mathrm{hc}} B S \cos (\varphi)$,

where $S$ is the section of the core turns (equal to $d^{2}$ ) and $\mu_{\text {app-hc }}$ is the apparent permeability of the helical ferromagnetic core.

This apparent permeability is directly derived from the formula of the diabolo core, given in Eq. (4). In that case $N_{z}\left(m^{\prime}\right)$ is the demagnetizing coefficient for the cylinder of lengthto-diameter ratio $m^{\prime}=L / D$ and $\frac{d^{2}}{\pi D^{2} / 4}$ is the surface ratio between the square section of the core turns and the end discs' section of the core.

Finally, the sensitivity of the induction sensor, assuming low-frequency operation $\left(\omega \ll \omega_{0}\right)$, is obtained:

$|T(j \omega)|=\left|\frac{e_{\mathrm{hc}}}{B}\right|=\omega N n \mu_{\mathrm{app}-\mathrm{hc}} S \cos (\varphi)$.

The resistance of the coil $\left(R_{\mathrm{hc}}\right)$ is a derivation of the Eq. (1), while the inductance formula is intuitively obtained:

$\mathrm{E}_{\mathrm{hc}}=\lambda(n N)^{2} \mu_{0} \frac{\mu_{\mathrm{app}-\mathrm{hc}} S \cos (\varphi)}{l}$.

The NEMI of the orthogonal induction sensor can be estimated using Eq. (20).

$\mathrm{NEMI}_{\mathrm{hc}}=\frac{\sqrt{4 k T R_{\mathrm{hc}}+e_{\mathrm{PA}}^{2}}}{\omega N n \mu_{\mathrm{app}-\mathrm{hc}} S \cos (\varphi)}$.

\subsection{Experimental results}

By using the previous set of equations we have determined the number of turns of an orthogonal sensor to get the same NEMI as the diabolo core sensor designed within the context of the BepiColombo space mission (namely $2 \mathrm{pT} / \sqrt{(\mathrm{Hz})}$ at $10 \mathrm{~Hz}$ from Coillot et al., 2010). The following set of design parameters was chosen: $d_{\mathrm{w}}=140 \mu \mathrm{m}, L_{\mathrm{c}}=100 \mathrm{~mm}, n=28$, $d=3 \mathrm{~mm}, D_{\mathrm{O}}=20 \mathrm{~mm}, a=3.5 \mathrm{~mm}$ and $L_{\mathrm{w}}=90 \mathrm{~mm}$. The design result led to a 400-turn coil on a single layer. The

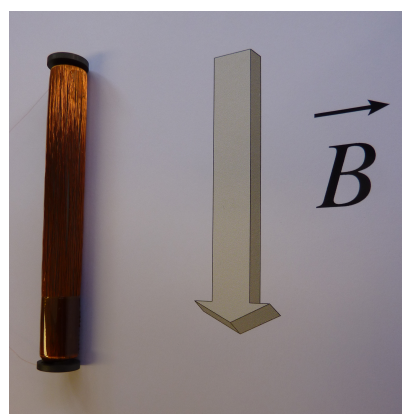

Figure 5. Picture of the orthogonal induction sensor prototype.

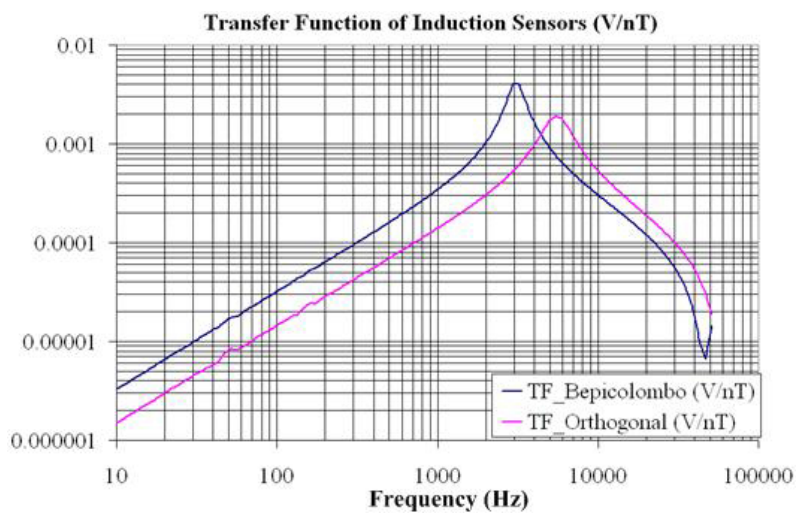

Figure 6. Transfer function of the orthogonal induction sensor (pink curve) versus that of the BepiColombo sensor (blue curve).

expected sensitivity at $10 \mathrm{~Hz}$ is about $2300 \mathrm{~V} / \mathrm{T}$, while the expected resistance should be four times lower than the one of the BepiColombo sensor (cf. Table 2). The orthogonal induction sensor prototype is shown in Fig. 5. It can be noticed that the coil normal direction is orthogonal to the direction of the magnetic field.

The advantage of the helicore is that the sensitivity criterion can be met with a small number of turns; the drawback is the difficulty in winding the core.

The transfer function reported in Fig. 6 demonstrates that the orthogonal induction sensor is able to efficiently measure magnetic fields. Its transfer function is compared to the BepiColombo induction sensor (blue curve), which uses many more turns (14000 turns for the BepiColombo one versus 400 turns for the orthogonal one). The sensitivities at $10 \mathrm{~Hz}$ for the BepiColombo and the orthogonal induction sensors are 3200 and $1500 \mathrm{~V} / \mathrm{T}$ respectively. The sensitivity of the orthogonal induction sensor prototype is $35 \%$ lower than the expected one (cf. summary of performances in Table 2). A gap between the ferromagnetic end discs and the helicore part or a small crack in the core is suspected to explain the difference. Since the resistance of the orthogonal induction sensor prototype is four times lower than that of the BepiColombo one, it implies that the signal-to-noise ratio (SNR) of the two sensors is comparable. An advantage of 


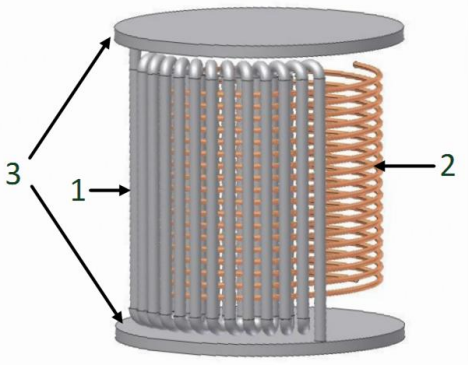

Figure 7. Diagram of the coiled-core induction sensor. (1) shows the ferromagnetic coil, (2) the conductive coil and (3) the ferromagnetic discs.

the orthogonal induction sensor is the higher resonance frequency, which permits extension of the frequency range of the measurement. The helical angle could reduce the performance, and thinner cores (on the helicore part) would reduce this angle and consequently increase the sensitivity. Lastly, the resonance frequency is lower than expected, which indicates that some leakage flux between core turns occurs. The use of a ferromagnetic wire to design an orthogonal induction sensor could solve the main problems encountered with the ferrite core.

\section{Coiled-core induction sensor}

\subsection{Description of the sensor configuration}

The coiled-core induction sensor, presented in Fig. 7, consists of an $n$-turns ferromagnetic wire (part 1) coiled around an $n_{\text {coil }}$-turns winding (part 2) made of conductive material like copper or aluminium. Similarly to the classical insulated conductive wire used to make the classical winding, the ferromagnetic wire should be insulated to leave a space between turns as to avoid a short-circuited magnetic path.

A ferromagnetic disc (part 3), acting as magnetic concentrators, is mounted at each end of the ferromagnetic wire in order to canalize the magnetic field. As a result, the magnetic field "flows" through the ferromagnetic wire and each conductive coil turn "sees" $n$ times the derivative of the flux of the ferromagnetic coil turns. The advantage of such a sensor is quite obvious, but we propose a modelling attempt to convince the reader of the potential interest of this theoretical sensor.

\subsection{Modelling of the coiled-core induction sensor}

Let us consider a copper winding with $N$ turns wound on a diameter $D_{\text {coil }}$ made with a conductive wire of resistivity $\rho$ and diameter $d_{\mathrm{w}}$ on a length $L_{\mathrm{w}}$. Similarly to the resistance formula of the induction sensor (cf. Eq. 1), the winding re- sistance of the coiled-core $\left(R_{\mathrm{cc}}\right)$ sensor is expressed as

$R_{\mathrm{cc}}=4 \rho N \frac{\left(D_{\text {coil }}+N\left(d_{\mathrm{w}}+2 t\right)^{2} / L_{\mathrm{w}}\right)}{d_{\mathrm{w}}^{2}}$.

On the one hand, the available area to coil the ferromagnetic wire is given by

$S_{\text {coil }}=\frac{\pi D_{\text {coil }}^{2}}{4}$

On the other hand the core section for a single wire is given by

$S_{\text {core }}=\frac{\pi d^{2}}{4}$

For a given core-coil filling factor $k_{\mathrm{f}}$ (a value of $\simeq 0.9$ is considered for the design example), the coil-core number $(n)$ is deduced:

$n=\frac{k_{\mathrm{f}} S_{\text {coil }}}{S_{\text {core }}}$.

In this relation we assume that the cored coil could be distributed on many layers inside the coil winding area. Let us now consider the sensor length $L_{\mathrm{c}}$ and the diameter of the ferromagnetic discs $D_{\mathrm{O}}$, the length-to-diameter ratio being $m^{\prime \prime}=L_{\mathrm{c}} / D_{\mathrm{O}}$. The apparent permeability, derived from Eq. (4), can be expressed as

$\mu_{\text {app-cc }}=\frac{\mu_{\mathrm{r}}}{1+N_{z}\left(m^{\prime \prime}\right) \frac{d^{2}}{D_{0}^{2}}\left(\mu_{\mathrm{r}}-1\right)}$.

The induced voltage modulus and the sensitivity of the induction sensor(assuming low-frequency operation $\left.\left(\omega \ll \omega_{0}\right)\right)$ are given by Eqs. (26) and (27) respectively:

$e_{\mathrm{cc}}=N n S_{\text {core }} \mu_{\text {app-cc }} \omega B$,

$|T(j \omega)|=\frac{e_{\mathrm{cc}}}{B}=N n S_{\text {core }} \mu_{\text {app-cc }} \omega$.

The NEMI equation for the coiled-core sensor is

$\mathrm{NEMI}_{\mathrm{cc}}=\frac{\sqrt{4 k T R_{\mathrm{cc}}+e_{\mathrm{PA}}^{2}}}{N n S_{\text {core }} \mu_{\mathrm{app}-\mathrm{cc}} \omega}$.

Similarly to the orthogonal induction sensor, a design attempt is performed (we choose the following set of design parameters: $d_{\mathrm{w}}=70 \mu \mathrm{m}, L=20 \mathrm{~mm}, d=1 \mathrm{~mm}, D_{\mathrm{O}}=20 \mathrm{~mm}$ and $L_{\mathrm{w}}=18 \mathrm{~mm}$ ). The electronic amplifier noise parameters $\left(e_{\mathrm{PA}}\right.$ and $\left.i_{\mathrm{PA}}\right)$ remain identical to previous cases. Because of the compactness of the coiled-core sensor $\left(L=D_{\mathrm{O}} \Rightarrow\right.$ $m^{\prime \prime} \approx 1$ ), a demagnetizing factor $N_{z}\left(m^{\prime \prime}\right)=1 / 3$ was considered (similarly to the value usually considered for sphere or cube). The resolution of the induction sensor design problem (i.e. number of turns verifying NEMI $=2 \mathrm{pT} / \sqrt{(\mathrm{Hz})}$ at $10 \mathrm{~Hz}$, using Eq. 28) lead to the parameters given in Table 2. 
Table 2. Design parameters for diabolo, orthogonal and coiled-core induction sensors for an equal NEMI goal: $2 \mathrm{pT} / \sqrt{(\mathrm{Hz})}$ at $10 \mathrm{~Hz}$, assuming $e_{\mathrm{PA}}=4 \mathrm{nV} / \sqrt{(\mathrm{Hz})}$ and $i_{\mathrm{PA}}=20 \mathrm{fA} / \sqrt{(\mathrm{Hz})}$.

\begin{tabular}{llll}
\hline Parameters & Diabolo & Ortho. & Coiled core \\
\hline Sensor length $\left(L_{\mathrm{c}}\right.$ in $\left.\mathrm{mm}\right)$ & 100 & 100 & 20 \\
\hline Winding length $\left(L_{\mathrm{w}}\right.$ in $\left.\mathrm{mm}\right)$ & 90 & 90 & 18 \\
\hline Core diameter/side $(d$ in $\mathrm{mm})$ & 4 & 3 & 1 \\
\hline Pitch $(2 \pi b$ in $\mathrm{mm})$ & N/A & 3.5 & N/A \\
\hline Helix radius $(a$ in mm $)$ & N/A & 5 & N/A \\
\hline End diameter $\left(D_{\mathrm{O}}\right.$ in mm) & 14 & 20 & 20 \\
\hline Coil diameter $\left(D_{\mathrm{coil}}\right.$ in mm $)$ & N/A & N/A & 10 \\
\hline Copper wire turns $(N)$ & 15600 & 400 & 465 \\
\hline Core wire turns $(n)$ & N/A & 28 & 90 \\
\hline Wire diameter $\left(d_{\mathrm{w}}\right.$ in $\left.\mu \mathrm{m}\right)$ & 70 & 140 & 70 \\
\hline Resistance $(R$ in $\Omega)$ & 1260 & 377 & 200 \\
\hline Apparent permeability & 295 & 377 & 1070 \\
\hline Sensitivity $(\mathrm{V} / \mathrm{T})$ & 3260 & 2360 & 2200 \\
\hline
\end{tabular}

This solution, which remains theoretical, could permit for strong reduction in the size of the sensor for a given sensor sensitivity. For the considered design, it suggests that a coiled-core induction sensor could be five times smaller than a classical induction sensor. The availability of windable ferromagnetic wire is the weakness of this conceptual sensor.

\section{Conclusions}

The three induction sensors reported in this work offer new possibilities for improvements. We believe that the coiledcore induction sensor is the most promising one even if it remains theoretical as the prototype has not been built. Its manufacturing is strongly dependent on the availability of coilable and insulated ferromagnetic wire. Nevertheless, the orthogonal induction sensor and the coiled-core one are similar. The orthogonal induction sensor prototype has allowed for confirming the predicted performance, which provides good confidence concerning the real wound-core sensor performance. These three induction sensors could be adapted to enhance other magnetic sensors, especially fluxgates and giant magneto-impedance (GMI). For instance, in the case of the GMI, the excitation current could flow through the ferromagnetic wire, while the conductive winding could be used as a pick-up coil. The closeness of the ferromagnetic wire could enhance the skin effect by means of the proximity effect, which could permit having high GMI ratio even at low frequency.
Acknowledgements. The authors would like to thank CNES (Centre National d'Etudes Spatiales), which has funded the prototype manufacturing within the context of a spacecraft mission study.

Edited by: B. Jakoby

Reviewed by: two anonymous referees

\section{References}

Bozorth. R. M. and Chapin, D.: Demagnetizing factors of rods, J. Appl. Phys., 13, 320-327, 1942.

Coillot, C. and Leroy, P.: Induction Magnetometers: Principle, Modeling and Ways of Improvement, Magnetic Sensors - Principles and Applications, edited by: Kuang, K., ISBN: 978-953-510232-8, InTech, 2012.

Coillot, C., Moutoussamy, J., Leroy, P., Chanteur, G., and Roux, A.: Improvements on the design of search coil magnetometer for space experiments, Sens. Lett., 5, 167-170 2007.

Coillot, C., Moutoussamy, J., Lebourgeois, R., Ruocco, S., and Chanteur, G.: Principle and performance of a dual-band search coil magnetometer: A new instrument to investigate fluctuating magnetic fields in space, IEEE Sens. J., 10, 255-260, 2010.

Dupuis, J. C.: Optimization of a 3-AXIS Induction Magnetometer for Airbone Geophysical Exploration, Master of Science in Egineering, University of New Brunswick, 2003.

Grosz, A. and Paperno, E.: Analytical Optimization of LowFrequency Search Coil Magnetometers, IEEE Sens. J., 12, 27192723, 2012.

Grosz, A., Paperno, E., Amrusi, S., and Liverts, E.: Integration of the electronics and batteries inside the hollow core of a search coil, J. App. Phys., 107, 09E703-E709E703-3, 2010.

Korepanov, V. and Pronenko, V.: Induction Magnetometers - Design Peculiarities, Sensors and Transducers Journal, 120, 92106, 2010.

Lichtenberger, J., Ferencz, C., Bodnar, L., Hamar, D., and Steinbach, P.: Automatic whistler detector and analyzer system, J. Geophys. Res., 113, 2156-2202, 2008.

Lukoschus, D.: Optimization theory for induction-coil magnetometers at higher frequencies, IEEE T. Geosci. Elect., GE-17, 56-63, 1979.

Osborn, J. A.: Demagnetizing factors of the general ellipsoids, 67, 351-357, 1945.

Ozaki, M., Yagitani, S., Takahashi, K., and Nagano, I.: Development of a new portable Lightning Location System, IEICE Trans. Commun., E95-B, No. 1, January 2012.

Ozaki, M., Yagitani, S., Takahashi, K., and Nagano, I.: DualResonant Search Coil for Natural Electromagnetic Waves in the Near-Earth Environment, IEEE Sens. J., 13, 644-650, 2013.

Prance, R. J., Clarck, T. D., and Prance, H.: Ultra low noise induction magnetometer for variable temperature operation, Sensor. Actuator., 85, 361-364, 2000.

Rhouni, A., Sou, G., Leroy, P., and Coillot, C.: A Very Low 1/f Noise and Radiation-Hardened CMOS Preamplifier for High Sensitivity Search Coil Magnetometers, IEEE Sens. J., 13, 159166, 2012.

Ripka, P.: Magnetic sensors and magnetometers, Ed. Artech House, 2000.

Roux, A., Le Contel, O., Coillot, C., Bouabdellah, A., de la Porte, B., Alison, D., Ruocco, R., and Vassal, M. C.: The search coil 
magnetometer for THEMIS, Space Sci. Rev., 141, 265-275, 2008.

Seran, H. C. and Fergeau, P.: An optimized low frequency three axis search coil for space research, Rev. Sci. Instrum., 76, 0445020044502-9, 2005.

Shimin, F., Suihua, Z., and Zhiyi, C.: A very low noise preamplifier for extremely low frequency magnetic antenna, Journal of Semiconductors, 34, 075003-075003-5, 2013.
Sudduth, K. A., Drummond, S. T., and Kitchen, N. R.: Accuracy issues in electromagnetic induction sensing of soil electrical conductivity for precision agriculture, Comput. Electron. Agr., 31, 239-264, 2001.

Tumanski, S.: Induction coil sensors - A review, Meas Sci. Technol., 18, R31-R46, 2007.

Yan, B.: An Optimization Method for Induction Magnetometer of $0.1 \mathrm{mHz}$ to $1 \mathrm{kHz}$, IEEE T. Magn., 49, 5294-5300, 2013. 